\title{
YIA-6
}

IUPHAR Young Investigator Award Competition

\section{Alcohol-induced hepatocyte pyroptosis results from miR-148a dysregulation through FoxO1}

\author{
Mi Jeong Heo, Tae Hyun Kim, Sang Geon Kim \\ College of Pharmarcy, Seoul National University, Korea
}

Alcoholic liver disease (ALD) is a leading cause of death among chronic liver diseases. MicroRNAs (miRNAs) are key contributors to the progression of liver diseases. Here, we investigated the role of forkhead box protein O1 (FoxO1) in alcohol-inducible hepatocyte death and explored the responsible microRNAs (miRNAs). In hepatocytes and animal models, alcohol treatment suppressed FoxO1. The miRNA microarray profile and qRT-PCR analyses enabled us to find substantial decrease of miR-148a in the liver of patients with alcoholic hepatitis. In mice subjected to Lieber-DeCarli alcohol liquid diet or binge alcohol drinking, miR-148a levels were markedly diminished. The levels of FoxO1 were significantly lowered in the patient specimens, correlating with miR-148a levels. FoxO1 was identified as a transcription factor for MIR148A transactivation. Treatment of hepatocytes with ethanol resulted in hepatocyte pyroptosis, which was reversed by miR-148a overexpression. Consequently, hepatocyte-specific delivery of miR-148a to mice attenuated alcohol-induced liver injury. Our results show that alcohol markedly decreases miR-148a expression in hepatocytes through FoxO1, facilitating NLRP3 inflammasome activation, which induces hepatocyte pyroptosis. Our findings provide information on novel targets for reducing incidence and progression of ALD. 